\title{
OPEN Statin Use and In-hospital Mortality in Patients with COVID-19 and Coronary Heart Disease
}

\author{
Lan Shen ${ }^{1,6}$, Lin Qiu ${ }^{2,6}$, Li Wang ${ }^{3,6}$, Hengye Huang ${ }^{4,6}$, Dong Liu², Ying Xiao², Yi Liu², \\ Jingjin Jin ${ }^{2}$, Xiulan $\mathrm{Liu}^{2}$, Dao Wen Wang ${ }^{5}$, Ben He ${ }^{1 \bowtie}$ \& Ning Zhou ${ }^{5 \bowtie}$
}

The worsening progress of coronavirus disease 2019 (COVID-19) is attributed to the proinflammatory state, leading to increased mortality. Statin works with its anti-inflammatory effects and may attenuate the worsening of COVID-19. COVID-19 patients were retrospectively enrolled from two academic hospitals in Wuhan, China, from 01/26/2020 to 03/26/2020. Adjusted in-hospital mortality was compared between the statin and the non-statin group by $\mathrm{CHD}$ status using multivariable Cox regression model after propensity score matching. Our study included 3133 COVID-19 patients (median age: $62 y$, female: $49.8 \%$ ), and $404(12.9 \%)$ received statin. Compared with the non-statin group, the statin group was older, more likely to have comorbidities but with a lower level of inflammatory markers. The Statin group also had a lower adjusted mortality risk (6.44\% vs. $10.88 \%$; adjusted hazard ratio [HR] $0.47 ; 95 \% \mathrm{Cl}, 0.29-0.77$ ). Subgroup analysis of CHD patients showed a similar result. Propensity score matching showed an overall $87 \%(\mathrm{HR}, 0.13 ; 95 \% \mathrm{Cl}, 0.05-0.36)$ lower risk of in-hospital mortality for statin users than nonusers. Such survival benefit of statin was obvious both among $\mathrm{CHD}$ and non-CHD patients (HR $=0.30$ [0.09-0.98]; HR $=0.23$ [0.1-0.49], respectively). Statin use was associated with reduced in-hospital mortality in COVID-19. The benefit of statin was both prominent among CHD and non-CHD patients. These findings may further reemphasize the continuation of statins in patients with CHD during the COVID-19 era.

Syndrome of acute respiratory stress coronavirus 2 (SARS-COV-2) has infected more than 200 million cases, with more than 4.26 million deaths has been reported ${ }^{1}$. The severity of COVID-19 is attributed to the inflammatory status, presented as an increased inflammatory biomarker such as C-Reactive Protein (CRP), Interleukin 2 (IL-2), and Interleukin 6 (IL-6), which are predictors of mortality ${ }^{2}$. Statin, a well-known anti-inflammatory medication, has been suggested as the possible medication to attenuate the worsening inflammatory reactions during COVID $-19^{3}$. However, animal studies showed that statin increases membrane-bound angiotensin-converting enzyme two receptors, the port of entry for COVID-19 virus. Clinicians are also concerned about the assumption that statin may enhance the virus entry into the human cells ${ }^{4,5}$. Consequently, there are fears that patients may discontinue statin during COVID-19 treatment.

Pre-existing coronary heart disease (CHD) predisposes patients to susceptibility to COVID-19 and serves as one of the strongest predictors in COVID-19 mortality ${ }^{6}$. Cardiomyocyte are specific target for SARS-CoV-2 ${ }^{7}$. SARS-CoV-2 infected the cardiomyocyte directly by virus interaction with angiotensin-converting enzyme 2 (ACE2) receptor which was activated by transmembrane protease serine protease-2 (TMPRSS2) ${ }^{8}$, enhanced virus's entrance and replication $^{9-11}$. Moreover, common comorbidity of CHD such as diabetes increases overinflammation, inflammatory/oxidative stress, cytokine storm following virus entrance and replication, and the extent of cytokine storm was associated with disease severity ${ }^{12-14}$. Previous studies showed that statin protected diabetic COVID-19 patients from in-hospital mortality by anti-inflammatory properties and antiplatelet effect ${ }^{3}$. CHD is highly prevalent in COVID-19 patients, especially severe patients ${ }^{15}$. Statin was the most widely used

\footnotetext{
${ }^{1}$ Department of Cardiology, Clinical Research Unit, Shanghai Chest Hospital, Shanghai Jiaotong University, Shanghai 200030, China. ${ }^{2}$ Department of Pharmacy, Tongji Hospital, Tongji Medical College, Huazhong University of Science and Technology, 1095 Jiefang Ave., Wuhan 430030, China. ${ }^{3}$ Department of Geriatrics, School of Medicine, Shanghai Renji Hospital, Shanghai Jiaotong University, Shanghai 200127, China. ${ }^{4}$ School of Public Health, School of Medicine, Shanghai Jiaotong University, Shanghai 200025, China. ${ }^{5}$ Division of Cardiology, Department of Internal Medicine, Tongji Hospital, Tongji Medical College, Huazhong University of Science and Technology, 1095 Jiefang Ave, Wuhan 430030, China. ${ }^{6}$ These authors contributed equally: Lan Shen, Lin Qiu, Li Wang and Hengye Huang. ${ }^{\square}$ email: heben241@126.com; zhouning@tjh.tjmu.edu.cn
} 
medication among CHD patients and is well known for its anti-inflammatory effect and effect in reducing reactive oxygen species and platelet reactivity. Our study sought to determine if statin use is associated with in-hospital survival benefits among COVID-19, especially in CHD patients.

\section{Material and methods}

Study design and participants. This retrospective, multi-center study included 3133 patients with COVID-19. All patients were admitted to two academic hospitals, the Sino-French new city branch and the Optical Valley branch affiliated with Tongji Hospital, Tongji Medical College, Huazhong University of Science and Technology. Both centers were exclusively managed COVID-19 patients in Wuhan, China. Tongji Hospital Ethics Commission approved the study. Written informed consent was waived because of the retrospective nature of the study by the Ethical Committee of Tongji Hospital, Tongji Medical College, Huazhong University of Science and Technology.

Inclusion criteria were patients who were 18 years of age or older, with COVID-19 admitted from January 26 to April 30, 2020. According to the New Coronavirus Pneumonia Prevention and Control Program (7th edition) published by the National Health Commission of China and WHO guidance, the diagnosis of COVID-19 was confirmed by qualitative Reverse Transcription Polymerase Chain Reaction (RT-RNA) tests on a nose/ throat swab positive for COVID-191,16. Patients with incomplete medical records (e.g., transfer to other hospitals) were excluded from the analysis. Patients with contraindications for statins use, including presented serum levels of CK or aminotransferase of more than five times the upper limit of normal (ULN) at admission, were also excluded.

Data collection. Electronic medical records of hospitalized patients with COVID-19 were reviewed to extract information on demographic information, history of comorbidities, clinical characteristics, laboratory data on admission, seven days post-admission and their peak value, therapeutic interventions (antiplatelet therapy, beta-blockers, angiotensin-converting enzyme inhibitors [ACEI] and ARBs [ARB], Calcium Channel Blocker [CCB], antiviral treatment, immunological treatment, ventilation, and Extracorporeal membrane oxygenation $[\mathrm{ECMO}]$ ), vasopressor medication such as dopamine, epinephrine, and norepinephrine during the hospitalization, and in-hospital mortality. The patient demographic information (age and sex), laboratory data on admission (blood cell count, D-Dimer, organ function markers, serum electrolyte, and inflammatory marker Interleukin-6 [IL-6], C-Reactive Protein [CRP], Procalcitonin [PCT]), laboratory data seven days post-admission and their peak value were collected from the laboratory information system. Comorbidities were extracted from medical history. The symptom's time to hospital presentation, length of stay, and in-hospital treatments were collected from medical records. All data were double-checked against source documents by two independent researchers.

Definition. The severity of COVID-19 was classified according to the diagnosis and treatment scheme for COVID-19 of China (7th edition) $)^{16}$. CHD patients were defined as those who had a medical record shown CHD as one of the comorbidities in the discharge diagnosis by clinicians. Patients were classified as statin group if medical history showed statin administration within the first three days post-hospital admission.

Statistical analysis. Continuous variables were expressed as the median and interquartile range (IQR), and categorical variables were expressed as number and percentage (\%). Statistical differences between the two groups were analyzed by the Mann-Whitney U test for continuous variables, while categorical variables were compared by Fisher's exact test or $\chi 2$ test. The risk of in-hospital mortality and the corresponding hazards ratio (HR) were calculated using the multivariable Cox regression model to compare the statin group versus the nonstatin group. A full model included demographics, clinical and laboratory variables independently associated with statin use or in-hospital mortality at a $\mathrm{P}<0.05$. The final multivariable adjustment including statin use (yes or no), age, sex, history of comorbidities, respiratory rate, severity classification, Lab results including serum B-type natriuretic peptide (BNP), Troponin I (TNI), aspartate aminotransferase (AST), Alanine transaminase (ALT), Creatinine (Cr), K, Na, Ca, glucose, Albumin, Lactate dehydrogenase (LDH), White blood cell (WBC), Lymphocyte, Neutrocyte, Platelet, CRP (C Reactive Protein), Procalcitonin (PCT), Interleukin 6 (IL-6), Interleukin 2 (IL-2), Tumor necrosis factor- $\alpha$ (TNF- $\alpha$ ), activated partial thromboplastin time (APTT), international normalized ratio (INR), D-Dimer and Total Cholesterol (TC), vasoactive drugs, ARB and beta-blocker (BB) use during hospitalization. A two-side a less than 0.05 was considered statistically different. Data were analyzed in R-3.6.3 (R Foundation for Statistical Computing, Vienna, Austria) and SPSS Statistics (version 22.0, IBM, Armonk, NY, USA).

Propensity score-matched analysis. To minimize baseline differences between statin and non-statin groups, we performed propensity score-matched analysis (PSM). Baseline matching variables included age, sex, history of comorbidities, Na, Albumin, Glucose, Hemoglobin, Lymphocyte, Platelet, CRP, IL-6, TC, serum troponin I (TNI) levels, severity classification, use of vasoactive medicine, antiplatelet therapy, ACEI, ARB, betablocker, and CCB. To further balance the baseline difference between statin and non-statin groups in subgroups of the CHD and non-CHD populations, we performed PSM again in the CHD and non-CHD groups, respectively. Residual imbalance $(\mathrm{p}<0.05)$ between statin and the non-statin group was further adjusted in the Cox regression model.

Statin users and nonusers were paired at 1:1 according to the propensity scores using exact matching with a caliper size of 0.05 . The balance of covariates was evaluated by p-value before and after matching, and $\mathrm{p}$-value $>=0.05$ was considered qualified balancing between the two groups. For subgroup study of CHD or nonCHD population, the pair ratio was also 1:1 between statin and non-statin group, with the caliper size as 0.05. 


\section{Result}

1. Patient Characteristics

Overall, 3133 patients ( $62 \pm 19$ years old; $49.8 \%$ female) were admitted with COVID-19. CHD (7.9\%), Diabetes mellitus (13.2\%), hypertension (317\%), and Chronic obstructive pulmonary (6.4\%) were highly prevalent comorbidities. On average, patients presented $13(\mathrm{IQR}, 12)$ days after the onset of symptoms and had elevated inflammatory markers on admission including CRP (11.5; IQR, $56.9 \mathrm{mg} / \mathrm{dL})$, IL-6 (5.0; IQR, $19.5 \mathrm{ng} / \mathrm{mL}$ ) and PCT (0.06; IQR, $0.07 \mathrm{ng} / \mathrm{mL}$ ) (Table 1).

2. Statin Use and In-Hospital Mortality

Among all admitted patients, $12.9 \%(\mathrm{n}=404)$ received a statin. Those on statin therapy were older $(67 \pm 15$ vs. $61 \pm 20$ years; $\mathrm{p}<0.001$ ) and had more likely to have comorbidities such as CHD, diabetes, cerebrovascular disease, dyslipidemia, hypertension, heart failure, and atrial fibrillation. (All $p<0.001$ ) Despite a higher comorbidity burden, we observed lower inflammatory markers such as at presentation a lower IL-2 (523.5, IQR [422.25] vs. 583.5, IQR [579.25], $\mathrm{p}=0.001)$, seven days later a lower IL-2 (431, IQR [328.5] vs. 504.5, IQR [443.5], $\mathrm{p}<0.001)$ and lower IL-2 peak level (551.5, IQR [431.75] vs. 606, IQR [630.75], p<0.001). Also, patients on a statin had a similar D-Dimer to start with but a lower D-Dimer 7 days later $(0.49$, IQR [0.8] vs. 0.63 , IQR [1.35], $\mathrm{p}=0.006$ ). (Table 1).

Meanwhile, we observed lower cumulative in-hospital mortality in those who were receiving a statin (6.44\% versus $10.88 \%$; $\mathrm{P}<0.01$, unadjusted HR $0.46[0.31,0.69])$. Further analysis revealed that this effect was prominent both in CHD and non-CHD patients. Patients on a statin had a lower cumulative in-hospital mortality both with $\mathrm{CHD}(10.4 \%$ versus $26 \%$; $\mathrm{p}=0.001$; ) or without $\mathrm{CHD}(4.7 \%$ versus $10.2 \% ; \mathrm{p}=0.003)$. (Table 2).

In the logistic regression model, after adjusting for age, sex, comorbidities, lab results on admission, including inflammatory markers, in-hospital medications, and severity classification, the use of statin was associated with lower all-cause mortality (adjusted HR, 0.47; 95\% CI, 0.29-0.77) versus non-statin group. The results remained consistent and statistically significant with a propensity score matching analysis, demonstrating a lower risk of in-hospital mortality in the statin group (adjusted HR, 0.13; 95\% CI [0.05-0.36]). (Table 2).

3. CHD and Statin Use

Among patients with CHD, 125 (50.4\%) received, and 123 (49.6\%) did not receive a statin. Those on a statin were at a similar age, with the similar possibility to have comorbidities such as hypertension, dyslipidemia, heart failure, diabetes, and pulmonary disease, as well as a similar COVID-19 severity classification. However, patients on statin were more likely to have concomitant use of aspirin, clopidogrel, beta-blocker, $\mathrm{ARB}$, and $\mathrm{CCB}($ all $\mathrm{p}<0.05)$. As for in-hospital treatment, those on statin were more likely to have concomitant use of aspirin, clopidogrel, beta-blocker, ARB, and CCB, but less likely to use vasoactive drugs (12 [9.6\%] vs. $23[18.7 \%$ ], all $\mathrm{p}<0.05)$. For inflammatory markers, those receiving a statin had a similar CRP but lower IL-6 $(8.35$, IQR [21.15] vs. 10.89, IQR [28.09], $\mathrm{p}=0.036)$ at presentation. Seven days later, patients on statin had both lower CRP (2.7, IQR [13.6] vs. 4.6, IQR [53.9], $\mathrm{p}=0.005)$ and lower IL-6 (4.96, IQR [11.08] vs. 9.5, IQR [40.74], $\mathrm{p}=0.023)$. When examining the peak level of CRP and IL-6, patients on a statin had a similar CRP level and a lower IL-6 level (10.52, IQR [29.49] vs. 11.69, IQR [47.46], p=0.02), compared with those off statin. Also, patients on statin had a similar D-Dimer at presentation but a lower D-Dimer 7 days later $(0.49$, IQR [0.8] vs. 0.63 , IQR [1.35], 0.006). (Table 3)

4. Statin Use and In-Hospital Mortality in CHD patients.

There was a 54\% reduction in in-hospital mortality (unadjusted HR 0.28, 95\% CI 0.15-0.54; adjusted HR $0.46,95 \%$ CI 0.23-0.90) associated with patients on statin with CHD admitted with COVID-19 in the multivariable risk regression model. Through PSM, statin recipients and nonrecipients had a well-balanced baseline characteristic, including age, sex, comorbidities, lab results, concomitant medications, and severity classification. (Table 4) The result remained statistically significant after PSM, and statin was associated with reduced in-hospital mortality both in CHD (adjusted HR 0.30, 95\%CI 0.09-0.98) and non-CHD patients (adjusted HR 0.23, 95\%CI 0.1-0.49). (Table 3).

\section{Discussion}

In this retrospective study, statin use was associated with a lower risk of all-cause in-hospital mortality than the non-statin group among COVID-19 patients. The benefit was significant both among CHD patients and non$\mathrm{CHD}$ patients. Although unmeasured confounding and indication bias may have contributed to the observed protective association, these data showed that statin use was associated with survival benefits in COVID-19. These findings provide additional clinical evidence supporting continuous statin use in CHD patients with COVID-19, both in CHD and non-CHD patients. Given this study's retrospective nature, these data need further validation in randomized controlled trials to determine the efficacy of statin use in patients and COVID-19.

Due to the conflicting considerations of statin use during COVID-19, several studies examined statin use during COVID-19. A study in China showed a survival benefit in statin users vs. non-statin users ${ }^{17}$. A most recent study examined diabetes patients with COVID-19 and showed that statin use was associated with reduced in-hospital mortality from COVID-19 in diabetes mellitus patients ${ }^{3}$. Such benefits did not show in non-diabetic patients. In diabetes or hyperglycemic status, virus entrance and replication are intensified. COVID-19 leads to inflammatory/oxidative stress and exaggerate cytokine storm, which is particularly enhanced in diabetes ${ }^{12-14}$. In COVID-19, serum concentrations of proinflammatory cytokines, including IL-2, IL-6, and tumor necrosis factor (TNF)-a, were markedly increased in severe cases than moderate cases, suggesting that cytokine storms 


\begin{tabular}{|c|c|c|c|c|c|c|c|c|}
\hline & \multicolumn{4}{|c|}{ Before PSM $(\mathrm{N}=3133)$} & \multicolumn{4}{|c|}{ After PSM $(\mathrm{N}=604)$} \\
\hline & All & \begin{tabular}{|l} 
Statin \\
$(\mathrm{N}=404)$
\end{tabular} & $\begin{array}{l}\text { Non-Statin } \\
(\mathrm{N}=2729)\end{array}$ & $\mathbf{p}$ & All & \begin{tabular}{|l} 
Statin \\
$(\mathrm{N}=302)$
\end{tabular} & $\begin{array}{l}\text { Non-Statin } \\
(\mathrm{N}=302)\end{array}$ & $\mathbf{p}$ \\
\hline Age (years) & $62(19)$ & $67(15)$ & $61(20)$ & $<0.001$ & $66(16)$ & $66(15.25)$ & $65(17)$ & 0.264 \\
\hline \multicolumn{9}{|l|}{ Sex (\%) } \\
\hline Male & $1572(50.18 \%)$ & $212(52.48 \%)$ & $1360(49.84 \%)$ & \multirow{2}{*}{0.322} & $312(51.66 \%)$ & $164(54.3 \%)$ & $148(49.01 \%)$ & \multirow{2}{*}{0.193} \\
\hline Female & $1561(49.82 \%)$ & $192(47.52 \%)$ & $1369(50.16 \%)$ & & $292(48.34 \%)$ & $138(45.7 \%)$ & $154(50.99 \%)$ & \\
\hline \multicolumn{9}{|c|}{ Comorbidities (\%) } \\
\hline Hypertension & $1159(36.99 \%)$ & $234(57.92 \%)$ & $925(33.9 \%)$ & $<0.001$ & $293(48.51 \%)$ & $158(52.32 \%)$ & $135(44.7 \%)$ & 0.061 \\
\hline Dyslipidemia & $23(0.73 \%)$ & $12(2.97 \%)$ & $11(0.4 \%)$ & $<0.001$ & $13(2.15 \%)$ & $5(1.66 \%)$ & $8(2.65 \%)$ & 0.401 \\
\hline $\mathrm{CHD}$ & $248(7.92 \%)$ & $125(30.94 \%)$ & $123(4.51 \%)$ & $<0.001$ & $102(16.89 \%)$ & $62(20.53 \%)$ & $40(13.25 \%)$ & 0.017 \\
\hline $\begin{array}{l}\text { Aortic Dissec- } \\
\text { tion }\end{array}$ & $4(0.13 \%)$ & $2(0.5 \%)$ & $2(0.07 \%)$ & 0.027 & $4(0.66 \%)$ & $2(0.66 \%)$ & $2(0.66 \%)$ & 1 \\
\hline Heart Failure & $33(1.05 \%)$ & $13(3.22 \%)$ & $20(0.73 \%)$ & $<0.001$ & $12(1.99 \%)$ & $6(1.99 \%)$ & $6(1.99 \%)$ & 1 \\
\hline $\begin{array}{l}\text { Atrial fibril- } \\
\text { lation }\end{array}$ & $20(0.64 \%)$ & $9(2.23 \%)$ & $11(0.4 \%)$ & $<0.001$ & $11(1.82 \%)$ & $5(1.66 \%)$ & $6(1.99 \%)$ & 0.761 \\
\hline $\begin{array}{l}\text { Other arrhyth- } \\
\text { mia }\end{array}$ & $333(10.63 \%)$ & $46(11.39 \%)$ & $287(10.52 \%)$ & 0.597 & $56(9.27 \%)$ & $37(12.25 \%)$ & $19(6.29 \%)$ & 0.012 \\
\hline $\begin{array}{l}\text { Diabetes } \\
\text { milieus }\end{array}$ & $414(13.21 \%)$ & $105(25.99 \%)$ & $309(11.32 \%)$ & $<0.001$ & $131(21.69 \%)$ & $65(21.52 \%)$ & $66(21.85 \%)$ & 0.922 \\
\hline Lung disease & $199(6.35 \%)$ & $27(6.68 \%)$ & $172(6.3 \%)$ & 0.77 & $41(6.79 \%)$ & $17(5.63 \%)$ & $24(7.95 \%)$ & 0.258 \\
\hline $\begin{array}{l}\text { Renal insuf- } \\
\text { ficiency }\end{array}$ & $69(2.2 \%)$ & $12(2.97 \%)$ & 57 (2.09\%) & 0.26 & $20(3.31 \%)$ & $10(3.31 \%)$ & $10(3.31 \%)$ & 1 \\
\hline Stroke & $102(3.26 \%)$ & $38(9.41 \%)$ & $64(2.35 \%)$ & $<0.001$ & $41(6.79 \%)$ & $20(6.62 \%)$ & $21(6.95 \%)$ & 0.872 \\
\hline Tumor & $129(4.12 \%)$ & $13(3.22 \%)$ & $116(4.25 \%)$ & 0.33 & $23(3.81 \%)$ & $10(3.31 \%)$ & $13(4.3 \%)$ & 0.524 \\
\hline Liver disease & $55(1.76 \%)$ & $2(0.5 \%)$ & $53(1.94 \%)$ & 0.039 & $5(0.83 \%)$ & $2(0.66 \%)$ & $3(0.99 \%)$ & 0.654 \\
\hline \multicolumn{9}{|c|}{ Lab on admission } \\
\hline $\begin{array}{l}\text { BNP } \\
(\mathrm{pg} / \mathrm{ml})\end{array}$ & $85(327)$ & $118.5(640.75)$ & $82(289)$ & 0.248 & $99(459.25)$ & $93(491)$ & $130(433)$ & 0.819 \\
\hline $\begin{array}{l}\text { Hs-cTnI (ng/ } \\
\mathrm{ml} \text { ) }\end{array}$ & $4(6.6)$ & $6.15(7.18)$ & $3.7(6.2)$ & 0.459 & $5.3(7.2)$ & $5(7.1)$ & $5.55(7.43)$ & 0.051 \\
\hline $\operatorname{ALT}(\mathrm{u} / \mathrm{l})$ & $23(24)$ & $24(21)$ & $22(24)$ & 0.546 & $24(23.75)$ & $24(22)$ & $23(27)$ & 0.71 \\
\hline AST (u/l) & $25(19)$ & $25(16)$ & $25(19)$ & 0.312 & $25(18)$ & $24.5(16)$ & $25(20)$ & 0.244 \\
\hline $\begin{array}{l}\text { Creatinine } \\
(\mu \mathrm{mol} / \mathrm{l})\end{array}$ & $68(27)$ & $70(26)$ & $68(28)$ & 0.714 & $69(26)$ & $69(26)$ & $69(26)$ & 0.298 \\
\hline $\mathrm{K}(\mathrm{mmol} / \mathrm{L})$ & $4.19(0.66)$ & $4.19(0.77)$ & $4.19(0.64)$ & 0.296 & $4.17(0.72)$ & $4.23(0.8)$ & $4.16(0.67)$ & 0.571 \\
\hline $\mathrm{Na}(\mathrm{mmol} / \mathrm{L})$ & $139.9(4.2)$ & $139.55(5.07)$ & $139.9(4.1)$ & $<0.001$ & $139.6(4.6)$ & $139.6(5.1)$ & $139.55(4.03)$ & 0.525 \\
\hline $\mathrm{Ca}(\mathrm{mmol} / \mathrm{L})$ & $2.16(0.17)$ & $2.15(0.16)$ & $2.16(0.17)$ & 0.347 & $2.16(0.17)$ & $2.16(0.16)$ & $2.15(0.17)$ & 0.427 \\
\hline $\begin{array}{l}\begin{array}{l}\text { Glucose } \\
(\mathrm{mmol} / \mathrm{L})\end{array} \\
\end{array}$ & $5.91(2.37)$ & $6.17(2.75)$ & $5.87(2.27)$ & 0.001 & $6.09(2.46)$ & $6.06(2.53)$ & $6.11(2.23)$ & 0.836 \\
\hline ALB (g/L) & $36.3(8.3)$ & $35.6(7.58)$ & $36.4(8.4)$ & 0.07 & $35.5(7.68)$ & 35.9 (7.45) & $35.1(8)$ & 0.301 \\
\hline LDH (U/L) & 248 (143) & $258(129)$ & 247 (145) & 0.316 & $255(132.75)$ & 247 (114.25) & $260.5(161.25)$ & 0.165 \\
\hline $\mathrm{Hb}(\mathrm{g} / \mathrm{l})$ & $127(22)$ & $124(21)$ & $127(22)$ & 0.05 & $125(22)$ & $124(20.25)$ & $126(24)$ & 0.558 \\
\hline WBC $\left(\times 10^{9} / \mathrm{l}\right)$ & $5.84(3.01)$ & $6.08(2.91)$ & $5.81(2.98)$ & 0.195 & $6.03(2.9)$ & $6.06(2.88)$ & $6.01(2.73)$ & 0.458 \\
\hline Lymph $\left(\times 10^{9} / \mathrm{l}\right)$ & $1.2(0.87)$ & $1.19(0.8)$ & $1.2(0.88)$ & 0.381 & $1.2(0.84)$ & $1.25(0.82)$ & $1.12(0.85)$ & 0.074 \\
\hline $\operatorname{PLT}\left(\times 10^{9} / \mathrm{l}\right)$ & $221(117)$ & $226(135.5)$ & $220(115)$ & 0.013 & $226(129.75)$ & $229.5(131.5)$ & $225(126.25)$ & 0.477 \\
\hline hs_CRP (mg/l) & $11.5(56.9)$ & $12.9(51.53)$ & $11.3(57.3)$ & 0.353 & $14.15(57.15)$ & $11.15(53)$ & $19.2(62.83)$ & 0.406 \\
\hline IL-2 (pg/ml) & $526(488.5)$ & $526(424.5)$ & 525 (495.5) & 0.196 & $552.5(492)$ & $523.5(422.25)$ & $583.5(579.25)$ & 0.001 \\
\hline IL_6 (pg/ml) & $4.98(19.46)$ & $6.35(15.88)$ & $4.84(19.73)$ & 0.278 & $6.45(19)$ & $5.91(15.01)$ & $7.17(23.85)$ & 0.478 \\
\hline TNF- $\alpha(\mathrm{ng} / \mathrm{ml})$ & $8(4.5)$ & $8.3(4.3)$ & $7.9(4.5)$ & 0.586 & $8.3(4.35)$ & $8.1(4.25)$ & $8.6(4.43)$ & 0.101 \\
\hline PCT (ng/ml) & $0.06(0.07)$ & $0.06(0.06)$ & $0.06(0.07)$ & 0.245 & $0.07(0.07)$ & $0.06(0.06)$ & $0.07(0.08)$ & 0.91 \\
\hline $\operatorname{APTT}(\mathrm{s})$ & $38.6(6.5)$ & $38.5(6.38)$ & $38.7(6.5)$ & 0.103 & $38.6(6.65)$ & $38.55(6.35)$ & $38.7(7)$ & 0.761 \\
\hline PT_INR (s) & $1.06(0.12)$ & $1.05(0.13)$ & $1.06(0.12)$ & 0.379 & $1.06(0.13)$ & $1.05(0.13)$ & $1.06(0.12)$ & 0.283 \\
\hline $\begin{array}{l}\text { D_Dimer }(\mu \mathrm{g} / \\
\mathrm{ml})\end{array}$ & $0.67(1.42)$ & $0.78(1.41)$ & $0.66(1.4)$ & 0.85 & $0.78(1.58)$ & $0.69(1.4)$ & $0.87(1.71)$ & 0.509 \\
\hline $\mathrm{TC}(\mathrm{mmol} / \mathrm{L})$ & $3.82(1.25)$ & $3.71(1.5)$ & $3.83(1.23)$ & 0.224 & $3.77(1.36)$ & $3.78(1.51)$ & $3.77(1.23)$ & 0.26 \\
\hline \multicolumn{9}{|c|}{ 7-days after admission } \\
\hline APTT (s) & $38.2(6.2)$ & 38.5 (6.18) & $38.2(6.2)$ & 0.492 & $38.4(6.5)$ & 38.4 (6.23) & 38.4 (6.93) & 0.096 \\
\hline PT_INR (s) & $1.04(0.11)$ & $1.04(0.12)$ & $1.04(0.11)$ & 0.525 & $1.04(0.11)$ & $1.04(0.11)$ & $1.04(0.12)$ & 0.172 \\
\hline $\begin{array}{l}\text { D_Dimer ( } \mu \mathrm{g} / \\
\mathrm{ml})\end{array}$ & $0.52(1.02)$ & $0.56(0.96)$ & $0.51(1.04)$ & 0.047 & $0.56(1.02)$ & $0.49(0.8)$ & $0.63(1.35)$ & 0.006 \\
\hline hs_CRP (mg/l) & $2.2(8)$ & $2.2(8.1)$ & $2.2(8)$ & 0.032 & $2.2(6.75)$ & $1.95(5.75)$ & $2.4(9.15)$ & 0.021 \\
\hline
\end{tabular}




\begin{tabular}{|c|c|c|c|c|c|c|c|c|}
\hline & \multicolumn{4}{|c|}{ Before PSM $(\mathrm{N}=3133)$} & \multicolumn{4}{|c|}{ After PSM $(\mathrm{N}=604)$} \\
\hline & All & $\begin{array}{l}\text { Statin } \\
(\mathrm{N}=404)\end{array}$ & $\begin{array}{l}\text { Non-Statin } \\
(\mathrm{N}=2729)\end{array}$ & $\mathbf{p}$ & All & $\begin{array}{l}\text { Statin } \\
(\mathrm{N}=302)\end{array}$ & $\begin{array}{l}\text { Non-Statin } \\
(\mathrm{N}=302)\end{array}$ & $\mathbf{p}$ \\
\hline $\mathrm{IL}-2(\mathrm{pg} / \mathrm{ml})$ & $450(384)$ & $443.5(334.5)$ & $451(390)$ & 0.088 & $460(371.5)$ & $431(328.5)$ & $504.5(443.5)$ & $<0.001$ \\
\hline IL_6 (pg/ml) & $3.27(7.97)$ & $3.72(8.37)$ & $3.2(7.95)$ & 0.077 & $3.6(7.9)$ & $3.2(6.46)$ & $4.13(9.71)$ & 0.2 \\
\hline TNF- $\alpha$ (ng/ml) & $7.7(4.6)$ & $8.2(4.6)$ & $7.7(4.7)$ & 0.591 & $8.1(4.5)$ & $8.1(4.43)$ & $8.1(4.63)$ & 0.566 \\
\hline $\mathrm{TC}(\mathrm{mmol} / \mathrm{L})$ & $4.23(1.41)$ & $3.8(1.54)$ & $4.27(1.36)$ & $<0.001$ & $4.03(1.5)$ & $3.98(1.54)$ & $4.07(1.44)$ & 0.391 \\
\hline \multicolumn{9}{|l|}{ Lab peak } \\
\hline $\begin{array}{l}\text { IL6 peak (pg/ } \\
\mathrm{ml})\end{array}$ & $6.27(24.83)$ & $7.59(22.93)$ & $6.03(25.19)$ & 0.066 & $7.45(22.55)$ & $6.8(17.87)$ & $8.85(29.44)$ & 0.13 \\
\hline $\begin{array}{l}\text { IL2 peak (pg/ } \\
\mathrm{ml})\end{array}$ & $553(509)$ & $554(438.25)$ & $553(520.5)$ & 0.099 & $569.5(512.25)$ & $551.5(431.75)$ & $606(630.75)$ & $<0.001$ \\
\hline $\begin{array}{l}\text { TNF-apeak } \\
\text { (ng/ml) }\end{array}$ & $8.7(5)$ & $8.9(4.65)$ & $8.6(4.9)$ & 0.495 & $8.95(4.78)$ & $8.8(4.53)$ & $9(4.83)$ & 0.327 \\
\hline $\begin{array}{c}\text { CRP peak } \\
(\mathrm{mmol} / \mathrm{L})\end{array}$ & $14.2(61.55)$ & $16.6(58.68)$ & $13.9(62.5)$ & 0.397 & $17.55(61.8)$ & $14.25(58.38)$ & $22(67.78)$ & 0.359 \\
\hline \multicolumn{9}{|l|}{ Meds (\%) } \\
\hline $\begin{array}{l}\text { Norepineph- } \\
\text { rine }\end{array}$ & $237(7.56 \%)$ & $28(6.93 \%)$ & $209(7.66 \%)$ & 0.606 & $38(6.29 \%)$ & $12(3.97 \%)$ & $26(8.61 \%)$ & 0.019 \\
\hline Epinephrine & $342(10.92 \%)$ & $37(9.16 \%)$ & $305(11.18 \%)$ & 0.225 & $61(10.1 \%)$ & $18(5.96 \%)$ & $43(14.24 \%)$ & 0.001 \\
\hline Dopamine & $200(6.38 \%)$ & $25(6.19 \%)$ & $175(6.41 \%)$ & 0.863 & $34(5.63 \%)$ & $12(3.97 \%)$ & $22(7.28 \%)$ & 0.078 \\
\hline Dobutamine & $36(1.15 \%)$ & $5(1.24 \%)$ & $31(1.14 \%)$ & 0.858 & $10(1.66 \%)$ & $4(1.32 \%)$ & $6(1.99 \%)$ & 0.524 \\
\hline Aspirin & $269(8.59 \%)$ & $172(42.57 \%)$ & $97(3.55 \%)$ & $<0.001$ & $183(30.3 \%)$ & $89(29.47 \%)$ & $94(31.13 \%)$ & 0.659 \\
\hline Clopidogrel & $142(4.53 \%)$ & $101(25 \%)$ & $41(1.5 \%)$ & $<0.001$ & $87(14.4 \%)$ & $47(15.56 \%)$ & $40(13.25 \%)$ & 0.418 \\
\hline Ticagrelor & $14(0.45 \%)$ & $12(2.97 \%)$ & $2(0.07 \%)$ & $<0.001$ & $6(0.99 \%)$ & $4(1.32 \%)$ & $2(0.66 \%)$ & 0.413 \\
\hline $\begin{array}{l}\text { Methylpredni- } \\
\text { solone }\end{array}$ & $873(27.86 \%)$ & $103(25.5 \%)$ & $770(28.22 \%)$ & 0.255 & $158(26.16 \%)$ & 77 (25.5\%) & $81(26.82 \%)$ & 0.712 \\
\hline ACEI & $92(2.94 \%)$ & $35(8.66 \%)$ & $57(2.09 \%)$ & $<0.001$ & $33(5.46 \%)$ & $19(6.29 \%)$ & $14(4.64 \%)$ & 0.372 \\
\hline $\mathrm{ARB}$ & $348(11.11 \%)$ & $110(27.23 \%)$ & $238(8.72 \%)$ & $<0.001$ & $117(19.37 \%)$ & $66(21.85 \%)$ & $51(16.89 \%)$ & 0.123 \\
\hline Beta-blocker & $91(2.9 \%)$ & $35(8.66 \%)$ & $56(2.05 \%)$ & $<0.001$ & $23(3.81 \%)$ & $15(4.97 \%)$ & $8(2.65 \%)$ & 0.137 \\
\hline $\mathrm{CCB}$ & $415(13.25 \%)$ & $92(22.77 \%)$ & $323(11.84 \%)$ & $<0.001$ & $112(18.54 \%)$ & $61(20.2 \%)$ & $51(16.89 \%)$ & 0.296 \\
\hline $\begin{array}{l}\text { Non-invasive } \\
\text { ventilator (\%) }\end{array}$ & $375(11.97 \%)$ & $57(14.11 \%)$ & $318(11.65 \%)$ & 0.156 & $81(13.41 \%)$ & $40(13.25 \%)$ & $41(13.58 \%)$ & 0.905 \\
\hline $\begin{array}{l}\text { Invasive Venti- } \\
\text { lator (\%) }\end{array}$ & $285(9.1 \%)$ & $38(9.41 \%)$ & $247(9.05 \%)$ & 0.817 & $50(8.28 \%)$ & $27(8.94 \%)$ & $23(7.62 \%)$ & 0.556 \\
\hline ECMO (\%) & $27(0.86 \%)$ & $4(0.99 \%)$ & $23(0.84 \%)$ & 0.765 & $5(0.83 \%)$ & $3(0.99 \%)$ & $2(0.66 \%)$ & $\mid 0.654$ \\
\hline \multicolumn{9}{|l|}{ Type (\%) } \\
\hline 1 & $888(28.34 \%)$ & $69(17.08 \%)$ & $819(30.01 \%)$ & \multirow{3}{*}{$<0.001$} & $145(24.01 \%)$ & $58(19.21 \%)$ & $87(28.81 \%)$ & \multirow{3}{*}{0.272} \\
\hline 2 & $1175(37.5 \%)$ & $173(42.82 \%)$ & $1002(36.72 \%)$ & & $227(37.58 \%)$ & $132(43.71 \%)$ & $95(31.46 \%)$ & \\
\hline 3 & $1070(34.15 \%)$ & $162(40.1 \%)$ & 908 (33.27\%) & & $232(38.41 \%)$ & $112(37.09 \%)$ & $120(39.74 \%)$ & \\
\hline
\end{tabular}

Table 1. Baseline characteristics of the study population by statin use before and after PSM. PSM, propensity score match; CHD, coronary vascular disease; BNP, serum B-type natriuretic peptide; TNI, Troponin I; ALT, Alanine Aminotransferase; AST, Aspartate transaminase; Cr, Creatinine; ALB, Albumin; LDH, Lactate dehydrogenase; WBC, White blood cell; Lymph, Lymphocyte; Plt, Platelet; CRP, C Reactive Protein; IL-2, Interleukin 2; IL-6, Interleukin 6; TNF- $\alpha$, P.C.T., Procalcitonin; Tumor necrosis factor- $\alpha$; APTT, activated partial thromboplastin time; INR, international normalized ratio; TC, Total Cholesterol; ACEI, Angiotensinconverting-enzyme inhibitors; ARB, angiotensin receptor blockers; CCB, Calcium Channel Blocker; ECMO, Extracorporeal membrane oxygenation. Continuous variables are presented as median (IQR), categorical variables are presented as $\mathrm{n}$ percentage.

\begin{tabular}{|l|l|l|r|l|l|l|}
\hline \multirow{2}{*}{ Statin vs. non-statin } & \multicolumn{2}{|l|}{ Crude mortality rate } & Unmatched & Unmatched & Matched \\
\cline { 2 - 7 } & Statin & Non-statin & p & $\begin{array}{l}\text { Unadjusted HR.* (95\% CI) Statin } \\
\text { vs. Nonstatin }\end{array}$ & $\begin{array}{l}\text { Adjusted HR. (95\% CI) Statin vs. } \\
\text { Nonstatin }\end{array}$ & $\begin{array}{l}\text { Adjusted HR. (95\% CI) Statin vs. } \\
\text { Nonstatin }\end{array}$ \\
\hline Overall & $6.44 \%$ & $10.88 \%$ & $<0.001$ & $0.46(0.31,0.69)$ & $0.47(0.29,0.77)$ & $0.13(0.05,0.36)$ \\
\hline CHD & $13(10.4 \%)$ & $32(26.0 \%)$ & 0.001 & $0.28(0.15,0.54)$ & $0.46(0.23,0.90)$ & $0.30(0.09,0.98)$ \\
\hline Non-CHD & $13(4.7 \%)$ & $265(10.2 \%)$ & 0.003 & $0.35(0.20,0.62)$ & $0.25(0.13,0.47)$ & $0.23(0.1,0.49)$ \\
\hline
\end{tabular}

Table 2. In-Hospital mortality in CHD and Non-CHD patients before and after the PSM. ${ }^{*} \mathrm{HR}$, the hazard ratio of in-hospital mortality between statin and non-statin (reference). 


\begin{tabular}{|c|c|c|c|c|c|c|c|c|}
\hline & \multicolumn{4}{|c|}{ Non CHD $(\mathrm{N}=2885)$} & \multicolumn{4}{|l|}{ CHD $(\mathrm{N}=248)$} \\
\hline & All & $\begin{array}{l}\text { Statin } \\
(\mathbf{N}=279)\end{array}$ & $\begin{array}{l}\text { Non-Statin } \\
(\mathrm{N}=2606)\end{array}$ & $\mathbf{p}$ & All & \begin{tabular}{|l} 
Statin \\
$(\mathrm{N}=125)$
\end{tabular} & $\begin{array}{l}\text { Non-Statin } \\
(\mathrm{N}=123)\end{array}$ & $\mathbf{p}$ \\
\hline Age & $61(20)$ & $65(15)$ & $60(20)$ & $<0.001$ & $69.5(13)$ & $71(13)$ & $69(15)$ & 0.377 \\
\hline \multirow{2}{*}{ Sex } & $1463(50.7 \%)$ & $154(55.2 \%)$ & $1309(50.2 \%)$ & \multirow{2}{*}{0.115} & $109(44 \%)$ & $58(46.4 \%)$ & $51(41.5 \%)$ & \multirow{2}{*}{0.436} \\
\hline & $1422(49.3 \%)$ & $125(44.8 \%)$ & $1297(49.8 \%)$ & & $139(56 \%)$ & $67(53.6 \%)$ & $72(58.5 \%)$ & \\
\hline Hospital Stay & $19.53(18.8)$ & $24.89(25.14)$ & $18.86(18.1)$ & $<0.001$ & $21.48(19.76)$ & $25.36(24.87)$ & $18.03(15.66)$ & $<0.001$ \\
\hline \multicolumn{9}{|l|}{ Comorbidities } \\
\hline Hypertension & $978(33.9 \%)$ & $138(49.5 \%)$ & $840(32.2 \%)$ & $<0.001$ & $181(73 \%)$ & $96(76.8 \%)$ & $85(69.1 \%)$ & 0.174 \\
\hline Dyslipidemia & $22(0.8 \%)$ & $11(3.9 \%)$ & $11(0.4 \%)$ & $<0.001$ & $1(0.4 \%)$ & $1(0.8 \%)$ & $0(0 \%)$ & 0.322 \\
\hline $\begin{array}{l}\text { Aortic Dissec- } \\
\text { tion }\end{array}$ & $3(0.1 \%)$ & $1(0.4 \%)$ & $2(0.1 \%)$ & 0.165 & $1(0.4 \%)$ & $1(0.8 \%)$ & $0(0 \%)$ & 0.322 \\
\hline Heart Failure & $21(0.7 \%)$ & $5(1.8 \%)$ & $16(0.6 \%)$ & 0.028 & $12(4.8 \%)$ & $8(6.4 \%)$ & $4(3.3 \%)$ & 0.25 \\
\hline $\begin{array}{l}\text { Atrial fibril- } \\
\text { lation }\end{array}$ & $11(0.4 \%)$ & $3(1.1 \%)$ & $8(0.3 \%)$ & 0.048 & $9(3.6 \%)$ & $6(4.8 \%)$ & $3(2.4 \%)$ & 0.322 \\
\hline $\begin{array}{l}\text { Other arrhyth- } \\
\text { mia }\end{array}$ & $316(11 \%)$ & $38(13.6 \%)$ & 278 (10.7\%) & 0.134 & $17(6.9 \%)$ & $8(6.4 \%)$ & $9(7.3 \%)$ & 0.776 \\
\hline $\begin{array}{l}\text { Diabetes } \\
\text { milieus }\end{array}$ & $342(11.9 \%)$ & $67(24 \%)$ & $275(10.6 \%)$ & $<0.001$ & $72(29 \%)$ & $38(30.4 \%)$ & $34(27.6 \%)$ & 0.634 \\
\hline Lung disease & $174(6 \%)$ & $16(5.7 \%)$ & $158(6.1 \%)$ & 0.827 & $25(10.1 \%)$ & $11(8.8 \%)$ & $14(11.4 \%)$ & 0.501 \\
\hline $\begin{array}{l}\text { Renal insuf- } \\
\text { ficiency }\end{array}$ & $54(1.9 \%)$ & $8(2.9 \%)$ & $46(1.8 \%)$ & 0.197 & $15(6 \%)$ & $4(3.2 \%)$ & $11(8.9 \%)$ & 0.058 \\
\hline Stroke & $90(3.1 \%)$ & $30(10.8 \%)$ & $60(2.3 \%)$ & $<0.001$ & $12(4.8 \%)$ & $8(6.4 \%)$ & $4(3.3 \%)$ & 0.25 \\
\hline Tumor & $118(4.1 \%)$ & $7(2.5 \%)$ & $111(4.3 \%)$ & 0.161 & $11(4.4 \%)$ & $6(4.8 \%)$ & $5(4.1 \%)$ & 0.78 \\
\hline Liver disease & $52(1.8 \%)$ & $2(0.7 \%)$ & $50(1.9 \%)$ & 0.152 & $3(1.2 \%)$ & $0(0 \%)$ & $3(2.4 \%)$ & 0.08 \\
\hline \multicolumn{9}{|l|}{ Lab on admission } \\
\hline $\begin{array}{l}\text { BNP } \\
(\mathrm{pg} / \mathrm{ml})\end{array}$ & $81(277.5)$ & $91(398)$ & $80(265.25)$ & 0.826 & 326 (821.5) & $445(883)$ & 224 (789) & 0.855 \\
\hline $\begin{array}{l}\text { Hs-cTnI (ng/ } \\
\text { ml) }\end{array}$ & $3.8(6.2)$ & $5(7.1)$ & $3.6(5.93)$ & 0.675 & $8.1(20.58)$ & $8.1(14.25)$ & $8.2(48.5)$ & 0.181 \\
\hline $\operatorname{ALT}(\mathrm{u} / \mathrm{l})$ & $22(24)$ & $24(21)$ & $22(24)$ & 0.39 & $23(24)$ & $23(23.5)$ & $24(25)$ & 0.999 \\
\hline $\operatorname{AST}(\mathrm{u} / \mathrm{l})$ & $25(18)$ & $24(16)$ & $25(18.25)$ & 0.284 & 25 (24.75) & $26(17)$ & $25(27)$ & 0.248 \\
\hline $\begin{array}{l}\text { Creatinine } \\
(\mu \mathrm{mol} / \mathrm{l})\end{array}$ & $68(27)$ & $68(24)$ & $68(27)$ & 0.631 & $74(34)$ & $73(29)$ & $74(42)$ & 0.875 \\
\hline $\mathrm{K}(\mathrm{mmol} / \mathrm{L})$ & $4.19(0.64)$ & $4.21(0.75)$ & $4.18(0.64)$ & 0.665 & $4.2(0.78)$ & $4.16(0.83)$ & $4.25(0.76)$ & 0.137 \\
\hline $\mathrm{Na}(\mathrm{mmol} / \mathrm{L})$ & $139.9(4)$ & $139.6(4.8)$ & $139.9(4)$ & 0.027 & $139.8(5.07)$ & $138.9(6.25)$ & $140.4(4.6)$ & 0.007 \\
\hline $\mathrm{Ca}(\mathrm{mmol} / \mathrm{L})$ & $2.16(0.17)$ & $2.16(0.17)$ & $2.16(0.17)$ & 0.529 & $2.14(0.16)$ & $2.13(0.16)$ & $2.15(0.18)$ & 0.742 \\
\hline $\begin{array}{l}\text { Glucose } \\
(\mathrm{mmol} / \mathrm{L})\end{array}$ & $5.87(2.31)$ & $6.15(2.81)$ & $5.85(2.25)$ & 0.004 & $6.28(2.34)$ & $6.22(2.55)$ & $6.37(2.22)$ & 0.924 \\
\hline ALB (g/L) & $36.4(8.4)$ & $35.6(7.5)$ & $36.5(8.4)$ & 0.122 & 35 (7.38) & $35.5(7.5)$ & $34.3(7)$ & 0.137 \\
\hline $\mathrm{LDH}(\mathrm{U} / \mathrm{L})$ & $247(140.5)$ & $247(122)$ & $246(143)$ & 0.267 & $263.5(165.5)$ & $265(151.5)$ & $251(230)$ & 0.045 \\
\hline $\mathrm{Hb}(\mathrm{g} / \mathrm{l})$ & $127(22)$ & $124(21)$ & $127(22)$ & 0.072 & $125.5(24.75)$ & $125(21.5)$ & $127(27)$ & 0.818 \\
\hline WBC $\left(\times 10^{9} / \mathrm{l}\right)$ & $5.83(3)$ & $6.11(2.92)$ & $5.8(2.98)$ & 0.118 & $6.05(3.03)$ & $5.99(2.79)$ & $6.05(3.02)$ & 0.08 \\
\hline Lymph $\left(\times 10^{9} / \mathrm{l}\right)$ & $1.21(0.86)$ & $1.23(0.77)$ & $1.21(0.87)$ & 0.836 & $1.04(0.92)$ & $1.09(0.82)$ & $0.98(0.95)$ & 0.569 \\
\hline PLT $\left(\times 10^{9} / \mathrm{l}\right)$ & $222(116)$ & $232(130)$ & $220(114.25)$ & 0.005 & $214.5(131)$ & $214(144.5)$ & $215(119)$ & 0.327 \\
\hline hs_CRP (mg/l) & $11.1(57)$ & $10(51)$ & $11.1(57.33)$ & 0.348 & $15.7(55.4)$ & $16.9(53.6)$ & $14.2(55.7)$ & 0.349 \\
\hline IL_2 (pg/ml) & $519(477.5)$ & $501(403)$ & $520(495.25)$ & 0.053 & $609(563.75)$ & $570(503)$ & $653(664)$ & 0.059 \\
\hline IL_6 (pg/ml) & $4.79(19)$ & $5.95(14.88)$ & $4.71(19.59)$ & 0.614 & $9.42(22.95)$ & $8.35(21.15)$ & $10.89(28.09)$ & 0.036 \\
\hline TNF- $\alpha(\mathrm{ng} / \mathrm{ml})$ & $7.9(4.4)$ & $8.1(4.2)$ & $7.9(4.5)$ & 0.255 & $8.95(5.18)$ & $8.8(5.2)$ & $9.1(5.4)$ & 0.341 \\
\hline PCT (ng/ml) & $0.06(0.07)$ & $0.06(0.05)$ & $0.06(0.07)$ & 0.276 & $0.07(0.11)$ & $0.06(0.1)$ & $0.08(0.13)$ & 0.289 \\
\hline $\operatorname{APTT}(\mathrm{s})$ & $38.7(6.5)$ & $38.5(6)$ & $38.7(6.5)$ & 0.102 & $38.45(6.28)$ & $38.5(7.2)$ & $38.4(5.7)$ & 0.769 \\
\hline PT_INR (s) & $1.05(0.12)$ & $1.05(0.14)$ & $1.06(0.12)$ & 0.448 & $1.07(0.12)$ & $1.06(0.1)$ & $1.08(0.17)$ & 0.05 \\
\hline $\begin{array}{l}\text { D_Dimer ( } \mu \mathrm{g} / \\
\mathrm{ml})\end{array}$ & $0.64(1.38)$ & $0.73(1.4)$ & $0.64(1.35)$ & 0.764 & $0.93(1.76)$ & $0.83(1.43)$ & $1.02(2.14)$ & 0.051 \\
\hline $\mathrm{TC}(\mathrm{mmol} / \mathrm{L})$ & $3.85(1.24)$ & $3.9(1.48)$ & $3.85(1.2)$ & 0.051 & $3.38(1.27)$ & $3.23(1.04)$ & $3.56(1.52)$ & 0.036 \\
\hline \multicolumn{9}{|c|}{ 7-days after admission } \\
\hline $\operatorname{APTT}(\mathrm{s})$ & $38.2(6)$ & $38.1(5.7)$ & $38.2(6.1)$ & 0.215 & $38.55(7.1)$ & $39.1(7.1)$ & $38.4(7.4)$ & 0.656 \\
\hline PT_INR & $1.04(0.11)$ & $1.04(0.13)$ & $1.04(0.11)$ & 0.193 & $1.06(0.15)$ & $1.05(0.11)$ & $1.06(0.19)$ & 0.372 \\
\hline $\begin{array}{l}\text { D_Dimer ( } \mu \mathrm{g} / \\
\mathrm{ml})\end{array}$ & $0.49(0.97)$ & $0.51(0.82)$ & $0.49(0.99)$ & 0.028 & $0.79(1.73)$ & $0.61(1.37)$ & $0.96(2.3)$ & 0.004 \\
\hline hs_CRP (mg/l) & $2(7.4)$ & $1.9(5.3)$ & $2.1(7.53)$ & 0.008 & $3.75(28.23)$ & $2.7(13.6)$ & \begin{tabular}{|l|}
$4.6(53.9)$ \\
\end{tabular} & 0.005 \\
\hline IL_2 (pg/ml) & 443 (373) & $429(329)$ & 446 (384.75) & 0.011 & 540.5 (509.75) & $508(427.5)$ & $594(676)$ & 0.072 \\
\hline
\end{tabular}




\begin{tabular}{|c|c|c|c|c|c|c|c|c|}
\hline & \multicolumn{4}{|c|}{ Non CHD (N= 2885) } & \multicolumn{4}{|l|}{ CHD $(\mathrm{N}=248)$} \\
\hline & All & $\begin{array}{l}\text { Statin } \\
(\mathrm{N}=279)\end{array}$ & $\begin{array}{l}\text { Non-Statin } \\
(\mathrm{N}=2606)\end{array}$ & $\mathbf{p}$ & All & $\begin{array}{l}\text { Statin } \\
(\mathrm{N}=125)\end{array}$ & $\begin{array}{l}\text { Non-Statin } \\
(\mathrm{N}=123)\end{array}$ & $\mathbf{p}$ \\
\hline IL_6 (pg/ml) & $3.16(7.01)$ & $3.25(6.52)$ & $3.15(7.06)$ & 0.163 & $5.87(21.19)$ & $4.96(11.08)$ & $9.5(40.74)$ & 0.023 \\
\hline TNF- $\alpha(\mathrm{ng} / \mathrm{ml})$ & $7.7(4.55)$ & $8.1(4.5)$ & $7.6(4.5)$ & 0.63 & $8.65(6.08)$ & $8.8(4.95)$ & $8.4(7.7)$ & 0.16 \\
\hline TC (mmol/L) & $4.26(1.36)$ & $4.11(1.54)$ & $4.28(1.34)$ & 0.082 & $3.56(1.55)$ & $3.4(1.15)$ & $3.98(1.93)$ & 0.064 \\
\hline \multicolumn{9}{|l|}{\begin{tabular}{|l|} 
Lab Peak \\
\end{tabular}} \\
\hline IL2peak & $545(507)$ & $518(395)$ & 547.5 (514.75) & 0.011 & 657 (673.5) & 624 (573.5) & $679(804)$ & 0.118 \\
\hline IL6peak & $5.99(23.95)$ & $6.85(17.85)$ & $5.94(24.62)$ & 0.15 & $11.52(36.42)$ & $10.52(29.49)$ & $11.69(47.46)$ & 0.02 \\
\hline TNFapeak & $8.6(4.8)$ & $8.8(4.3)$ & $8.6(5)$ & 0.516 & $9.75(5.95)$ & 9.6 (4.95) & $9.9(6.7)$ & 0.143 \\
\hline \begin{tabular}{|l|} 
CRPpeak \\
\end{tabular} & $13.5(60.5)$ & $13.5(55.9)$ & $13.5(61.73)$ & 0.218 & $24(73.35)$ & \begin{tabular}{|l|}
$27(71.4)$ \\
\end{tabular} & $19.4(88.2)$ & 0.175 \\
\hline \multicolumn{9}{|l|}{ Medicine } \\
\hline Norepinephrine & $202(7 \%)$ & $16(5.7 \%)$ & $186(7.1 \%)$ & 0.383 & $35(14.1 \%)$ & $12(9.6 \%)$ & $23(18.7 \%)$ & 0.04 \\
\hline Epinephrine & $294(10.2 \%)$ & $19(6.8 \%)$ & $275(10.6 \%)$ & 0.05 & $48(19.4 \%)$ & $18(14.4 \%)$ & $30(24.4 \%)$ & 0.047 \\
\hline Dopamine & $171(5.9 \%)$ & $12(4.3 \%)$ & $159(6.1 \%)$ & 0.226 & $29(11.7 \%)$ & $13(10.4 \%)$ & $16(13 \%)$ & 0.525 \\
\hline Dobutamine & $33(1.1 \%)$ & $3(1.1 \%)$ & $30(1.2 \%)$ & 0.91 & $3(1.2 \%)$ & $2(1.6 \%)$ & $1(0.8 \%)$ & 0.573 \\
\hline Aspirin & $171(5.9 \%)$ & $98(35.1 \%)$ & $73(2.8 \%)$ & $<0.001$ & 98 (39.5\%) & $74(59.2 \%)$ & $24(19.5 \%)$ & $<0.001$ \\
\hline Clopidogrel & $73(2.5 \%)$ & $48(17.2 \%)$ & $25(1 \%)$ & $<0.001$ & $69(27.8 \%)$ & $53(42.4 \%)$ & $16(13 \%)$ & $<0.001$ \\
\hline \begin{tabular}{|l|} 
Ticagrelor \\
\end{tabular} & $5(0.2 \%)$ & $4(1.4 \%)$ & $1(0 \%)$ & $<0.001$ & $9(3.6 \%)$ & $8(6.4 \%)$ & $1(0.8 \%)$ & 0.019 \\
\hline $\begin{array}{l}\text { Methylpredni- } \\
\text { solone }\end{array}$ & $791(27.4 \%)$ & $68(24.4 \%)$ & $723(27.7 \%)$ & 0.23 & $82(33.1 \%)$ & $35(28 \%)$ & $47(38.2 \%)$ & 0.088 \\
\hline ACEI & $69(2.4 \%)$ & $20(7.2 \%)$ & 49 (1.9\%) & $<0.001$ & $23(9.3 \%)$ & $15(12 \%)$ & $8(6.5 \%)$ & \begin{tabular}{|l|}
0.137 \\
\end{tabular} \\
\hline ARB & $281(9.7 \%)$ & $63(22.6 \%)$ & $218(8.4 \%)$ & $<0.001$ & 67 (27\%) & $47(37.6 \%)$ & $20(16.3 \%)$ & $<0.001$ \\
\hline Beta-blocker & $61(2.1 \%)$ & $12(4.3 \%)$ & $49(1.9 \%)$ & 0.008 & $30(12.1 \%)$ & $23(18.4 \%)$ & $7(5.7 \%)$ & 0.002 \\
\hline CCB & 348 (12.1\%) & $51(18.3 \%)$ & 297 (11.4\%) & 0.001 & $67(27 \%)$ & $41(32.8 \%)$ & $26(21.1 \%)$ & 0.039 \\
\hline $\begin{array}{l}\text { Non-invasive } \\
\text { ventilator (\%) }\end{array}$ & $341(11.8 \%)$ & $39(14 \%)$ & $302(11.6 \%)$ & 0.24 & $34(13.7 \%)$ & $18(14.4 \%)$ & $16(13 \%)$ & 0.751 \\
\hline $\begin{array}{l}\text { Invasive Venti- } \\
\text { lator (\%) }\end{array}$ & $256(8.9 \%)$ & $26(9.3 \%)$ & $230(8.8 \%)$ & 0.783 & $29(11.7 \%)$ & $12(9.6 \%)$ & $17(13.8 \%)$ & 0.303 \\
\hline ECMO (\%) & $26(0.9 \%)$ & $3(1.1 \%)$ & $23(0.9 \%)$ & 0.746 & $1(0.4 \%)$ & $1(0.8 \%)$ & $0(0 \%)$ & 0.322 \\
\hline \multicolumn{9}{|l|}{\begin{tabular}{|l|} 
Type \\
\end{tabular}} \\
\hline 1 & $860(29.8 \%)$ & $58(20.8 \%)$ & $802(30.8 \%)$ & \multirow{3}{*}{0.004} & $28(11.3 \%)$ & $11(8.8 \%)$ & $17(13.8 \%)$ & \multirow{3}{*}{0.761} \\
\hline 2 & $1075(37.3 \%)$ & $118(42.3 \%)$ & 957 (36.7\%) & & $100(40.3 \%)$ & 55 (44\%) & $45(36.6 \%)$ & \\
\hline 3 & $950(32.9 \%)$ & $103(36.9 \%)$ & $847(32.5 \%)$ & & \begin{tabular}{|l|}
$120(48.4 \%)$ \\
\end{tabular} & $59(47.2 \%)$ & $61(49.6 \%)$ & \\
\hline
\end{tabular}

Table 3. Baseline characteristics of the study population by statin use in CHD or non-CHD population. CHD, coronary heart disease; BNP, serum B-type natriuretic peptide; TNI, Troponin I; ALT, Alanine Aminotransferase; AST, Aspartate transaminase; Cr, Creatinine; ALB, Albumin; LDH, Lactate dehydrogenase; WBC, White blood cell; Lymph, Lymphocyte; Plt, Platelet; CRP, C Reactive Protein; IL-2, Interleukin 2; IL-6, Interleukin 6; TNF- $\alpha$, P.C.T., Procalcitonin; Tumor necrosis factor- $\alpha$; APTT, activated partial thromboplastin time; INR, international normalized ratio; TC, Total Cholesterol; ACEI, Angiotensin-converting-enzyme inhibitors; ARB, angiotensin receptor blockers; CCB, Calcium Channel Blocker; ECMO, Extracorporeal membrane oxygenation. Continuous variables are presented as median (IQR), categorical variables are presented as $n$ percentage.

could be associated with disease severity. Statin was known for its anti-inflammatory quality. Diabetes and cardiovascular disease were two major comorbidities that increased the mortality of COVID-192,18,19, yet there is no study examining statin use in CHD patients. Also, our study examined the change of inflammatory marker levels at presentation, seven days post-admission, and their peak levels both in statin and non-statin groups among CHD patients.

CHD is one of the most prevalent comorbidities in COVID-19 patients. Our COVID-19 population has a CHD prevalence of 7.9\%, which echoes the previous COVID-19 report in Wuhan, and was lower than US studies $^{6}$. The definition of CHD in our study was those who had a medical record shown CHD as one of the discharge diagnoses. In such case, CHD included all patients with coronary heart disease, such as those who had a history of CHD, treated with PCI, CAGB, or receiving medical therapy, as long as clinicians have once diagnosed it. The individual case fatality rate of COVID-19 patients with CHD was $10.5 \%$, highest among those with any comorbidities, including chronic respiratory disease (6.3\%), cancer (5.6\%), diabetes (7.3\%), and hypertension $(6.0 \%)^{20}$. CHD predispose patients with proinflammatory and procoagulant status, together with COVID-19, increased the risk of thrombus formation, severe inflammatory response, and endothelial damage, leading to ACS, heart failure, cardiac arrhythmia, and sudden death ${ }^{21-23}$. COVID-19 can also directly trigger ACS by plaque rupture, coronary spasm, or microthrombi due to systemic inflammation or cytokine storm ${ }^{24}$. Meanwhile, the most common comorbidities associated with CHD, such as hypertension increased ACE2 receptor and facilitated 


\begin{tabular}{|c|c|c|c|c|c|c|c|c|}
\hline & \multicolumn{4}{|c|}{ Non-CHD $(\mathrm{N}=502)$} & \multicolumn{4}{|l|}{ CHD $(\mathrm{N}=102)$} \\
\hline & All & Statin $(\mathrm{N}=\mathbf{2 7 5})$ & $\begin{array}{l}\text { Non-Statin } \\
(\mathbf{N}=\mathbf{2 7 7})\end{array}$ & $\mathbf{p}$ & All & $\begin{array}{l}\text { Statin } \\
(\mathrm{N}=97)\end{array}$ & $\begin{array}{l}\text { Non-Statin } \\
(\mathrm{N}=95)\end{array}$ & $\mathbf{p}$ \\
\hline Age & $65(17)$ & $64(15)$ & $65(20)$ & 0.701 & $68.5(13.25)$ & $71(13)$ & $67(15.25)$ & 0.29 \\
\hline \multicolumn{9}{|l|}{ Sex } \\
\hline Male & $266(52.99 \%)$ & $132(55 \%)$ & $134(51.15 \%)$ & \multirow{2}{*}{0.388} & $46(45.1 \%)$ & $32(51.61 \%)$ & $14(35 \%)$ & \multirow{2}{*}{0.102} \\
\hline Female & $236(47.01 \%)$ & $108(45 \%)$ & $128(48.85 \%)$ & & $56(54.9 \%)$ & $30(48.39 \%)$ & $26(65 \%)$ & \\
\hline Length of stay & $21.69(21.01)$ & $24.02(25.55)$ & $20.33(19.38)$ & 0 & $22.02(23.17)$ & $26.51(25.84)$ & $18.35(15.74)$ & 0.016 \\
\hline \multicolumn{9}{|l|}{ Comorbidities } \\
\hline Hypertension & $217(43.23 \%)$ & $111(46.25 \%)$ & $106(40.46 \%)$ & 0.191 & $76(74.51 \%)$ & $47(75.81 \%)$ & $29(72.5 \%)$ & 0.712 \\
\hline Dyslipidemia & $13(2.59 \%)$ & $5(2.08 \%)$ & $8(3.05 \%)$ & 0.495 & $0(0 \%)$ & $0(0 \%)$ & $0(0 \%)$ & -- \\
\hline $\begin{array}{l}\text { Aortic Dissec- } \\
\text { tion }\end{array}$ & $3(0.6 \%)$ & $1(0.42 \%)$ & $2(0.76 \%)$ & 0.615 & $1(0.98 \%)$ & $1(1.61 \%)$ & $0(0 \%)$ & 0.425 \\
\hline Heart Failure & $9(1.79 \%)$ & $4(1.67 \%)$ & $5(1.91 \%)$ & 0.839 & $3(2.94 \%)$ & $2(3.23 \%)$ & $1(2.5 \%)$ & 0.834 \\
\hline $\begin{array}{l}\text { Atrial fibril- } \\
\text { lation }\end{array}$ & $6(1.2 \%)$ & $3(1.25 \%)$ & $3(1.15 \%)$ & 0.914 & $5(4.9 \%)$ & $2(3.23 \%)$ & $3(7.5 \%)$ & 0.334 \\
\hline $\begin{array}{l}\text { Other arrhyth- } \\
\text { mia }\end{array}$ & $51(10.16 \%)$ & $34(14.17 \%)$ & 17 (6.49\%) & 0.004 & $5(4.9 \%)$ & $3(4.84 \%)$ & $2(5 \%)$ & 0.971 \\
\hline $\begin{array}{l}\text { Diabetes } \\
\text { milieus }\end{array}$ & $100(19.92 \%)$ & $47(19.58 \%)$ & $53(20.23 \%)$ & 0.857 & $31(30.39 \%)$ & $18(29.03 \%)$ & $13(32.5 \%)$ & 0.713 \\
\hline Lung disease & $31(6.18 \%)$ & $11(4.58 \%)$ & $20(7.63 \%)$ & 0.157 & $10(9.8 \%)$ & $6(9.68 \%)$ & $4(10 \%)$ & 0.958 \\
\hline $\begin{array}{l}\text { Renal insuf- } \\
\text { ficiency }\end{array}$ & $15(2.99 \%)$ & $8(3.33 \%)$ & $7(2.67 \%)$ & 0.664 & $5(4.9 \%)$ & $2(3.23 \%)$ & $3(7.5 \%)$ & 0.334 \\
\hline Stroke & $37(7.37 \%)$ & $19(7.92 \%)$ & $18(6.87 \%)$ & 0.655 & $4(3.92 \%)$ & $1(1.61 \%)$ & $3(7.5 \%)$ & 0.137 \\
\hline Tumor & $17(3.39 \%)$ & $7(2.92 \%)$ & $10(3.82 \%)$ & 0.578 & $6(5.88 \%)$ & $3(4.84 \%)$ & $3(7.5 \%)$ & 0.581 \\
\hline Liver disease & $4(0.8 \%)$ & $2(0.83 \%)$ & $2(0.76 \%)$ & 0.93 & $1(0.98 \%)$ & $0(0 \%)$ & $1(2.5 \%)$ & 0.215 \\
\hline \multicolumn{9}{|c|}{ Lab on admission } \\
\hline BNP (pg/ml) & $97(345)$ & 85.5 (339) & $118.5(342.75)$ & 0.735 & 248.5 (759.25) & 315.5 (697) & 223.5 (795.5) & 0.876 \\
\hline $\begin{array}{l}\text { Hs-cTnI (ng/ } \\
\text { ml) }\end{array}$ & $4.8(7.13)$ & $4.7(6.68)$ & $5(7.43)$ & 0.145 & $7.95(14.45)$ & $7.55(7.7)$ & 8.15 (27.95) & 0.133 \\
\hline $\operatorname{ALT}(\mathrm{u} / \mathrm{l})$ & $24(24)$ & $24.5(21)$ & $22(26.5)$ & 0.582 & $25.5(26.25)$ & $23.5(25.5)$ & $26(29.25)$ & 0.816 \\
\hline $\operatorname{AST}(\mathrm{u} / \mathrm{l})$ & $25(19)$ & $24(16)$ & $25(20)$ & 0.174 & $25.5(20)$ & $25.5(16.75)$ & $25.5(19)$ & 0.814 \\
\hline $\begin{array}{l}\text { Creatinine } \\
(\mu \mathrm{mol} / \mathrm{l})\end{array}$ & $68(25.25)$ & $68.5(25.75)$ & $68(26)$ & 0.486 & $70(34.5)$ & $69(25.5)$ & $73.5(49)$ & 0.22 \\
\hline $\mathrm{K}(\mathrm{mmol} / \mathrm{L})$ & $4.19(0.71)$ & $4.23(0.79)$ & $4.16(0.67)$ & 0.633 & $4.09(0.82)$ & $4.19(0.9)$ & $3.97(0.65)$ & 0.744 \\
\hline $\mathrm{Na}(\mathrm{mmol} / \mathrm{L})$ & $139.6(4.43)$ & $139.8(4.75)$ & $139.5(4.2)$ & 0.879 & $139.4(5.68)$ & $139(6.55)$ & $140.35(4.32)$ & 0.428 \\
\hline $\mathrm{Ca}(\mathrm{mmol} / \mathrm{L})$ & $2.16(0.17)$ & $2.17(0.17)$ & $2.16(0.18)$ & 0.533 & $2.13(0.16)$ & $2.13(0.16)$ & $2.13(0.16)$ & 0.339 \\
\hline $\begin{array}{l}\text { Glucose } \\
(\mathrm{mmol} / \mathrm{L})\end{array}$ & $6.08(2.51)$ & $6.06(2.55)$ & $6.09(2.37)$ & 0.822 & $6.13(2.21)$ & $6.05(2.54)$ & $6.19(2.01)$ & 0.225 \\
\hline ALB (g/L) & $35.65(7.83)$ & $35.9(7.78)$ & $35.3(7.93)$ & 0.382 & $35.1(7.05)$ & $35.6(6.63)$ & $33.65(8.3)$ & 0.311 \\
\hline $\mathrm{LDH}(\mathrm{U} / \mathrm{L})$ & $256(130)$ & 241.5 (104.75) & 265 (158.5) & 0.071 & $248.5(168)$ & $263(168.25)$ & 225 (174.75) & 0.609 \\
\hline $\mathrm{Hb}(\mathrm{g} / \mathrm{l})$ & $126(22)$ & $125(20.75)$ & $126(24)$ & 0.79 & $124(24.25)$ & $123(23.25)$ & $128(25.75)$ & 0.548 \\
\hline WBC $\left(\times 10^{9} / 1\right)$ & $6.04(2.91)$ & $6.07(2.88)$ & $6.03(2.81)$ & 0.407 & $5.97(2.77)$ & $6.02(2.96)$ & $5.69(2.59)$ & 0.961 \\
\hline Lymph $\left(\times 10^{9} / \mathrm{l}\right)$ & $1.21(0.82)$ & $1.29(0.86)$ & $1.14(0.85)$ & 0.034 & $1.11(0.9)$ & $1.17(0.87)$ & $1.04(0.93)$ & 0.979 \\
\hline $\operatorname{PLT}\left(\times 10^{9} / \mathrm{l}\right)$ & 229.5 (128.5) & 231 (126.5) & $228.5(127.25)$ & 0.498 & $216.5(136.75)$ & $219(145)$ & 205 (107.75) & 0.582 \\
\hline hs_CRP (mg/l) & $13.6(58.83)$ & 8.95 (51.05) & $20.15(67.8)$ & 0.245 & $16.15(44.95)$ & $17(66.2)$ & $13.95(36.48)$ & 0.548 \\
\hline IL_2 (pg/ml) & $534.5(493.25)$ & 493 (408.75) & 566 (584.5) & 0 & $625.5(470.75)$ & $612(424.75)$ & $677.5(530.25)$ & 0.278 \\
\hline IL_6 (pg/ml) & $6(18.5)$ & $5.55(13.99)$ & $6.55(23.87)$ & 0.586 & $9.08(23.3)$ & $8.18(23.29)$ & $10.12(25.41)$ & 0.236 \\
\hline TNF- $\alpha(\mathrm{ng} / \mathrm{ml})$ & $8.2(4.3)$ & $8(4.18)$ & $8.5(4.33)$ & 0.042 & $9.25(4.9)$ & $8.9(5.2)$ & $9.85(4.58)$ & 0.789 \\
\hline PCT (ng/ml) & $0.07(0.07)$ & $0.06(0.05)$ & $0.07(0.07)$ & 0.886 & $0.07(0.09)$ & $0.06(0.1)$ & $0.08(0.09)$ & 0.956 \\
\hline APTT (s) & $38.7(6.8)$ & $38.5(6.1)$ & $38.75(7.33)$ & 0.294 & $38.5(6.8)$ & 38.75 (6.68) & $38.1(6.08)$ & 0.099 \\
\hline PT_INR (s) & $1.06(0.13)$ & $1.05(0.13)$ & $1.06(0.12)$ & 0.222 & $1.07(0.13)$ & $1.07(0.1)$ & $1.07(0.18)$ & 0.991 \\
\hline $\begin{array}{l}\text { D_Dimer }(\mu \mathrm{g} / \\
\mathrm{ml})\end{array}$ & $0.77(1.6)$ & $0.64(1.41)$ & $0.89(1.71)$ & 0.775 & $0.81(1.39)$ & $0.81(1.34)$ & $0.8(1.69)$ & 0.336 \\
\hline $\mathrm{TC}(\mathrm{mmol} / \mathrm{L})$ & $3.85(1.31)$ & $3.91(1.45)$ & $3.81(1.17)$ & 0.045 & $3.21(1.26)$ & $3.13(1.21)$ & 3.39 (1.67) & 0.527 \\
\hline \multicolumn{9}{|c|}{ 7-days after admission } \\
\hline APTT (s) & $38.3(6.3)$ & $38.1(5.98)$ & $38.4(6.9)$ & 0.073 & $38.8(6.9)$ & $38.95(6.45)$ & $38.4(9.4)$ & 0.959 \\
\hline PT_INR & $1.04(0.12)$ & $1.04(0.13)$ & $1.04(0.11)$ & 0.175 & $1.04(0.11)$ & $1.04(0.09)$ & $1.04(0.25)$ & 0.428 \\
\hline $\begin{array}{l}\text { D_Dimer }(\mu \mathrm{g} / \\
\mathrm{ml})\end{array}$ & $0.52(0.99)$ & $0.48(0.74)$ & $0.59(1.32)$ & 0.012 & $0.71(1.4)$ & $0.61(1.26)$ & $0.81(1.93)$ & 0.166 \\
\hline hs_CRP (mg/l) & $2.1(5.6)$ & $1.9(5.03)$ & $2.3(7.33)$ & 0.012 & $2.5(15.48)$ & $2.35(8.8)$ & $2.55(35.33)$ & 0.646 \\
\hline \multicolumn{9}{|l|}{ Continued } \\
\hline
\end{tabular}




\begin{tabular}{|c|c|c|c|c|c|c|c|c|}
\hline & \multicolumn{4}{|c|}{ Non-CHD $(\mathrm{N}=502)$} & \multicolumn{4}{|l|}{ CHD $(\mathrm{N}=102)$} \\
\hline & All & Statin $(N=275)$ & $\begin{array}{l}\text { Non-Statin } \\
(\mathrm{N}=277)\end{array}$ & $\mathbf{p}$ & All & $\begin{array}{l}\text { Statin } \\
(\mathrm{N}=97)\end{array}$ & $\begin{array}{l}\text { Non-Statin } \\
(\mathrm{N}=95)\end{array}$ & $\mathbf{p}$ \\
\hline $\mathrm{IL} \_2(\mathrm{pg} / \mathrm{ml})$ & $452(356.5)$ & $409(326.75)$ & $493.5(431.75)$ & 0 & $502.5(494.25)$ & $470(416.75)$ & $587(610)$ & 0.103 \\
\hline IL_6 (pg/ml) & $3.43(6.58)$ & $2.96(6.29)$ & $4.01(7.92)$ & 0.653 & $4.48(11.91)$ & $3.75(8.72)$ & $7.48(25.68)$ & 0.061 \\
\hline TNF- $\alpha(\mathrm{ng} / \mathrm{ml})$ & $8.05(4.2)$ & $8(4.28)$ & $8.1(4.2)$ & 0.774 & $9.45(5.65)$ & $9.15(5.4)$ & $9.45(7.05)$ & 0.246 \\
\hline $\mathrm{TC}(\mathrm{mmol} / \mathrm{L})$ & $4.12(1.54)$ & $4.11(1.61)$ & $4.14(1.53)$ & 0.741 & $3.52(1.32)$ & $3.46(1.23)$ & $3.88(1.44)$ & 0.586 \\
\hline \multicolumn{9}{|l|}{ Lab Peak } \\
\hline IL2peak & $551.5(510.75)$ & $514(414)$ & $596.5(629.75)$ & 0 & $654.5(591.75)$ & $637.5(491.75)$ & $702.5(769)$ & 0.317 \\
\hline IL6peak & $6.88(20.89)$ & $6.54(15.41)$ & $8.1(28)$ & 0.433 & $10.29(36.6)$ & $10.29(35.54)$ & $10.28(37.71)$ & 0.07 \\
\hline TNFapeak & $8.8(4.43)$ & $8.7(4)$ & $9(4.9)$ & 0.394 & $10.05(5.45)$ & $9.9(5.05)$ & $10.25(5.9)$ & 0.448 \\
\hline CRPpeak & $17.25(62)$ & $12.95(53.5)$ & $23.95(69.13)$ & 0.137 & $20.25(65.2)$ & $27.3(77.55)$ & $15.9(38.8)$ & 0.343 \\
\hline \multicolumn{9}{|l|}{ Medicine } \\
\hline Norepinephrine & $32(6.37 \%)$ & $11(4.58 \%)$ & $21(8.02 \%)$ & 0.116 & $6(5.88 \%)$ & $1(1.61 \%)$ & $5(12.5 \%)$ & 0.022 \\
\hline Epinephrine & $48(9.56 \%)$ & $13(5.42 \%)$ & $35(13.36 \%)$ & 0.002 & $13(12.75 \%)$ & $5(8.06 \%)$ & $8(20 \%)$ & 0.079 \\
\hline Dopamine & $27(5.38 \%)$ & $8(3.33 \%)$ & $19(7.25 \%)$ & 0.052 & $7(6.86 \%)$ & $4(6.45 \%)$ & $3(7.5 \%)$ & 0.84 \\
\hline Dobutamine & $9(1.79 \%)$ & $3(1.25 \%)$ & $6(2.29 \%)$ & 0.381 & $1(0.98 \%)$ & $1(1.61 \%)$ & $0(0 \%)$ & 0.425 \\
\hline Aspirin & $137(27.29 \%)$ & 66 (27.5\%) & $71(27.1 \%)$ & 0.92 & 46 (45.1\%) & $23(37.1 \%)$ & $23(57.5 \%)$ & 0.044 \\
\hline Clopidogrel & $50(9.96 \%)$ & $26(10.83 \%)$ & $24(9.16 \%)$ & 0.533 & $37(36.27 \%)$ & $21(33.87 \%)$ & $16(40 \%)$ & 0.534 \\
\hline Ticagrelor & $3(0.6 \%)$ & $2(0.83 \%)$ & $1(0.38 \%)$ & 0.513 & $3(2.94 \%)$ & $2(3.23 \%)$ & $1(2.5 \%)$ & 0.834 \\
\hline $\begin{array}{l}\text { Methylpredni- } \\
\text { solone }\end{array}$ & $126(25.1 \%)$ & $58(24.17 \%)$ & $68(25.95 \%)$ & 0.645 & $32(31.37 \%)$ & $19(30.65 \%)$ & $13(32.5 \%)$ & 0.846 \\
\hline ACEI & $22(4.38 \%)$ & $13(5.42 \%)$ & $9(3.44 \%)$ & 0.28 & $11(10.78 \%)$ & $6(9.68 \%)$ & $5(12.5 \%)$ & 0.657 \\
\hline ARB & $87(17.33 \%)$ & $46(19.17 \%)$ & $41(15.65 \%)$ & 0.299 & $30(29.41 \%)$ & $20(32.26 \%)$ & $10(25 \%)$ & 0.437 \\
\hline Beta-blocker & $13(2.59 \%)$ & $6(2.5 \%)$ & $7(2.67 \%)$ & 0.904 & $10(9.8 \%)$ & $9(14.52 \%)$ & $1(2.5 \%)$ & 0.047 \\
\hline $\mathrm{CCB}$ & $83(16.53 \%)$ & $40(16.67 \%)$ & $43(16.41 \%)$ & 0.939 & $29(28.43 \%)$ & $21(33.87 \%)$ & $8(20 \%)$ & 0.132 \\
\hline $\begin{array}{l}\text { Non-invasive } \\
\text { ventilator (\%) }\end{array}$ & $65(12.95 \%)$ & $33(13.75 \%)$ & $32(12.21 \%)$ & 0.609 & $16(15.69 \%)$ & $7(11.29 \%)$ & $9(22.5 \%)$ & 0.131 \\
\hline $\begin{array}{l}\text { Invasive Venti- } \\
\text { lator (\%) }\end{array}$ & $38(7.57 \%)$ & $21(8.75 \%)$ & 17 (6.49\%) & 0.34 & $12(11.76 \%)$ & $6(9.68 \%)$ & $6(15 \%)$ & 0.42 \\
\hline ECMO (\%) & $4(0.8 \%)$ & $2(0.83 \%)$ & $2(0.76 \%)$ & 0.93 & $1(0.98 \%)$ & $1(1.61 \%)$ & $0(0 \%)$ & 0.425 \\
\hline \multicolumn{9}{|l|}{ Type } \\
\hline 1 & $134(26.69 \%)$ & $53(22.08 \%)$ & $81(30.92 \%)$ & \multirow{3}{*}{0.421} & $11(10.78 \%)$ & $5(8.06 \%)$ & $6(15 \%)$ & \multirow{3}{*}{0.827} \\
\hline 2 & $182(36.25 \%)$ & $102(42.5 \%)$ & $80(30.53 \%)$ & & $45(44.12 \%)$ & $30(48.39 \%)$ & $15(37.5 \%)$ & \\
\hline 3 & $186(37.05 \%)$ & $85(35.42 \%)$ & $101(38.55 \%)$ & & $46(45.1 \%)$ & $27(43.55 \%)$ & $19(47.5 \%)$ & \\
\hline
\end{tabular}

Table 4. Baseline characteristics of the study population according to statin use in CHD or non-CHD population after PSM. CHD, coronary heart disease; BNP, serum B-type natriuretic peptide; TNI, Troponin I; ALT, Alanine Aminotransferase; AST, Aspartate transaminase; Cr, Creatinine; ALB, Albumin; LDH, Lactate dehydrogenase; WBC, White blood cell; Lymph, Lymphocyte; Plt, Platelet; CRP, C Reactive Protein; IL-2, Interleukin 2; IL-6, Interleukin 6; TNF- $\alpha$, P.C.T., Procalcitonin; Tumor necrosis factor- $\alpha$; APTT, activated partial thromboplastin time; INR, international normalized ratio; TC, Total Cholesterol; ACEI, Angiotensinconverting-enzyme inhibitors; ARB, angiotensin receptor blockers; CCB, Calcium Channel Blocker; ECMO, Extracorporeal membrane oxygenation. Continuous variables are presented as median (IQR), categorical variables are presented as $\mathrm{n}$ percentage.

virus entry, diabetes enhanced cytokine storm, and leads to multi-organ failure ${ }^{10,11,25}$. Moreover, COVID-19 was associated with hypercoagulation status and could cause increased intracoronary thrombosis and worsen STEMI outcomes ${ }^{26}$. Therefore, COVID-19 patients with CHD were four times more likely to die than average COVID-19 patients, with an elevated level of proinflammatory cytokines such as IL-6, CRP, and procoagulant factors such as D-Dimer ${ }^{20}$.

Statin is known for its anti-inflammatory and anticoagulant effects ${ }^{27}$. SARS-CoV-2 is targeting the endothelium, one of the largest organs in the human body. Notably, endothelial cells express all the co-factors necessary for the internalization of SARS-CoV-2 in human host cells ${ }^{28,29}$. Moreover, the most common comorbidities in COVID-19 such as hypertension and diabetes were generally predisposed with endothelial dysfunction, which promotes virus entry, replication, clotting cascade, microvascular obstruction and multi-organ failure ${ }^{25}$. Dysregulated RAS system in CHD patients also increases ACE2 expression, results in pre-existing endothelial dysfunction which facilitates virus entry, finally causees subsequent cardiac damages ${ }^{29-31}$. On the other hand, SARS-CoV-2 per se induces cytokine storm, which further exacerbates endothelial injury and dysfunction, ultimately leading to microvascular thrombi, pro-thrombotic state, and cardiovascular ischemia status ${ }^{25,29}$. Expert in bioengineering and nanomedicine have tried to develop numerous innovative pharmaceutical approaches targeting endothelial dysfunction to tackle this problem ${ }^{28}$. Statin is one of the most promising drug classes for 
treating endothelial dysfunction and preventing vascular damage in COVID-19. Similar to RAS inhibitors, statins improve endothelial function in patients with or at risk for cardiovascular disease. They improve endothelial function via reduction of LDL and suppression of pro-oxidant enzymes and other proinflammatory transcriptional and signal transduction pathways ${ }^{32}$. Previous studies showed that statin protected diabetic COVID-19 patients from in-hospital mortality by anti-inflammatory properties and antiplatelet effect ${ }^{3}$. Its acute administration improves survival in proinflammatory states such as myocardial infarction. Our study shows that statin use in CHD patients vastly improved in-hospital morality, with reduced IL-2, IL-6 level, and D-Dimer levels during the hospital stay.Similarly, such benefits are also seen in non-CHD patients and more critically ill patients. To avoid an unbalanced baseline, we used propensity score match to retest our result and yield a similar conclusion of statin benefit. However, due to this study's retrospective nature, a prospective design is warranted to prove the conclusion further.

\section{Limitation}

Our study should be viewed considering several limitations inherent in retrospective analyses. First, although we had detailed information on medical history, our observational study cannot exclude the potential impact of unmeasured confounders such as social status, the duration of statin use, and the reason for statin use. Second, we did not examine pre-hospital statin use. Such information was missed in the electronic data collection system because of the urgent COVID-19 time. However, we defined statin use as patients received statin within 3-day post-hospitalization. We choose this definition to include the statin group was patients who were regular statin users. Also, it has not been our routine practice for clinicians to initiate new statin therapy in patients with acute COVID-19 illness. In addition, clinical scenarios leading to statin cessation such as liver enzyme elevation were not significantly different between the statin and non-statin groups. Meanwhile, our data showed only two patients who initiated statin after three-day post-hospitalization. Both of them were fully recovered and discharged. As a result, excluding the two late-use statin cases may have underestimated the benefit of statin treatment on COVID-19 patients but would not change the direction of our results. Third, due to all patients were hospitalized with COVID-19 in Wuhan, generalizability may be limited. Finally, due to the observational study's inherent limitation, our study did not examine the causality between statin use and reduced in-hospital mortality, which should be tested only by a randomized control trial.

\section{Conclusion}

Among patients hospitalized with COVID-19, statin use was associated with reduced in-hospital mortality from COVID-19. The benefit of statin was both prominent among patients with CHD and non-CHD patients. These findings may further reemphasize the continuation of statins to patients with CHD during the COVID-19 era.

Ethical approval. All procedures performed in studies involving human participants were following the ethical standards of the institutional and/or national research committee and with the 1964 Helsinki declaration and its later amendments or comparable ethical standards.

Informed consent. This study was approved by the Ethics Commission of Tongji Hospital. Written informed consent was waived for the retrospective study by the Ethical Committee of Tongji Hospital, Tongji Medical College, Huazhong University of Science and Technology.

Consent for publication. All authors have also reviewed and approved the manuscript.

\section{Data availability}

The datasets generated during and/or analyzed during the current study are available from the corresponding author on reasonable request.

Received: 5 June 2021; Accepted: 30 August 2021

Published online: 13 December 2021

\section{References}

1. Organization., W. H. Coronavirus disease (COVID-19) pandemic, <https://covid19.who.int/> (2021).

2. Zhou, F. et al. Clinical course and risk factors for mortality of adult inpatients with COVID-19 in Wuhan, China: a retrospective cohort study. Lancet 395, 1054-1062. https://doi.org/10.1016/S0140-6736(20)30566-3 (2020).

3. Saeed, O. et al. Statin use and in-hospital mortality in patients with diabetes mellitus and COVID-19. J. Am. Heart Assoc. 9, e018475. https://doi.org/10.1161/JAHA.120.018475 (2020).

4. Tikoo, K. et al. Tissue specific up regulation of ACE2 in rabbit model of atherosclerosis by atorvastatin: Role of epigenetic histone modifications. Biochem. Pharmacol. 93, 343-351. https://doi.org/10.1016/j.bcp.2014.11.013 (2015).

5. South, A. M., Diz, D. I. \& Chappell, M. C. COVID-19, ACE2, and the cardiovascular consequences. Am. J. Physiol. Heart Circ. Physiol. 318, H1084-H1090. https://doi.org/10.1152/ajpheart.00217.2020 (2020).

6. Nishiga, M., Wang, D. W., Han, Y., Lewis, D. B. \& Wu, J. C. COVID-19 and cardiovascular disease: from basic mechanisms to clinical perspectives. Nat. Rev. Cardiol. 17, 543-558. https://doi.org/10.1038/s41569-020-0413-9 (2020).

7. D'Onofrio, N. et al. Glycated ACE2 receptor in diabetes: Open door for SARS-COV-2 entry in cardiomyocyte. Cardiovasc. Diabetol. 20, 99. https://doi.org/10.1186/s12933-021-01286-7 (2021).

8. Matarese, A., Gambardella, J., Sardu, C. \& Santulli, G. miR-98 regulates TMPRSS2 expression in human endothelial cells: Key implications for COVID-19. Biomedicines 8, 1. https://doi.org/10.3390/biomedicines8110462 (2020).

9. Deng, Q. et al. Suspected myocardial injury in patients with COVID-19: Evidence from front-line clinical observation in Wuhan, China. Int. J. Cardiol. 311, 116-121. https://doi.org/10.1016/j.ijcard.2020.03.087 (2020). 
10. Sardu, C. et al. Could anti-hypertensive drug therapy affect the clinical prognosis of hypertensive patients with COVID-19 infection? Data from centers of Southern Italy. J. Am. Heart Assoc. 9, e016948. https://doi.org/10.1161/JAHA.120.016948 (2020).

11. Sardu, C. et al. Implications of AB0 blood group in hypertensive patients with covid-19. BMC Cardiovasc. Disord. 20, 373. https:// doi.org/10.1186/s12872-020-01658-z (2020).

12. Sardu, C., Gargiulo, G., Esposito, G., Paolisso, G. \& Marfella, R. Impact of diabetes mellitus on clinical outcomes in patients affected by Covid-19. Cardiovasc. Diabetol. 19, 76. https://doi.org/10.1186/s12933-020-01047-y (2020).

13. Marfella, R. et al. Negative impact of hyperglycaemia on tocilizumab therapy in Covid-19 patients. Diabetes Metab. 46, 403-405. https://doi.org/10.1016/j.diabet.2020.05.005 (2020).

14. Sardu, C. et al. Hyperglycaemia on admission to hospital and COVID-19. Diabetologia 63, 2486-2487. https://doi.org/10.1007/ s00125-020-05216-2 (2020).

15. Clerkin, K. J. et al. COVID-19 and Cardiovascular Disease. Circulation 141, 1648-1655. https://doi.org/10.1161/CIRCULATIO NAHA.120.046941 (2020).

16. CNH, C. Chinese Clinical Guidance for COVID-19 Pneumonia Diagnosis and Treatment (7th edition), http://kjfy.meetingchina. org/msite/news/show/cn/3337.html (2020).

17. Zhang, X. J. et al. In-Hospital Use of Statins Is Associated with a Reduced Risk of Mortality among Individuals with COVID-19. Cell Metab 32, 176-187 e174, doi:https://doi.org/10.1016/j.cmet.2020.06.015 (2020).

18. Jordan, R. E., Adab, P. \& Cheng, K. K. Covid-19: risk factors for severe disease and death. BMJ 368, m1198. https://doi.org/10. 1136/bmj.m1198 (2020).

19. Ruan, Q., Yang, K., Wang, W., Jiang, L. \& Song, J. Clinical predictors of mortality due to COVID-19 based on an analysis of data of 150 patients from Wuhan, China. Intens. Care Med. 46, 846-848. https://doi.org/10.1007/s00134-020-05991-x (2020).

20. Vital surveillances: the epidemiological characteristics of an outbreak of 2019 novel coronavirus diseases (COVID-19) - China, 2020. China CDC Wkly 2, 113-122 (2020).

21. Fried, J. A. et al. The Variety of Cardiovascular Presentations of COVID-19. Circulation 141, 1930-1936. https://doi.org/10.1161/ CIRCULATIONAHA.120.047164 (2020).

22. Mehra, M. R. \& Ruschitzka, F. COVID-19 Illness and Heart Failure: A Missing Link?. JACC Heart Fail 8, 512-514. https://doi.org/ 10.1016/j.jchf.2020.03.004 (2020).

23. Varga, Z. et al. Endothelial cell infection and endotheliitis in COVID-19. Lancet 395, 1417-1418. https://doi.org/10.1016/S01406736(20)30937-5 (2020).

24. Libby, P., Tabas, I., Fredman, G. \& Fisher, E. A. Inflammation and its resolution as determinants of acute coronary syndromes. Circ. Res. 114, 1867-1879. https://doi.org/10.1161/CIRCRESAHA.114.302699 (2014).

25. Sardu, C. et al. Hypertension, thrombosis, kidney failure, and diabetes: Is COVID-19 an endothelial disease? A comprehensive evaluation of clinical and basic evidence. J. Clin. Med. 9, 1. https://doi.org/10.3390/jcm9051417 (2020).

26. Marfella, R. et al. SARS-COV-2 colonizes coronary thrombus and impairs heart microcirculation bed in asymptomatic SARSCoV-2 positive subjects with acute myocardial infarction. Crit. Care 25, 217. https://doi.org/10.1186/s13054-021-03643-0 (2021).

27. Sommeijer, D. W. et al. Anti-inflammatory and anticoagulant effects of pravastatin in patients with type 2 diabetes. Diabetes Care 27, 468-473. https://doi.org/10.2337/diacare.27.2.468 (2004).

28. Gambardella, J. \& Santulli, G. What is linking COVID-19 and endothelial dysfunction? Updates on nanomedicine and bioengineering from the 2020 AHA Scientific Sessions. Eur. Heart J. Cardiovasc. Pharmacother. 7, e2-e3. https://doi.org/10.1093/ehjcvp/ pvaa145 (2021).

29. Kaur, S., Tripathi, D. M. \& Yadav, A. The Enigma of Endothelium in COVID-19. Front. Physiol. 11, 989. https://doi.org/10.3389/ fphys.2020.00989 (2020).

30. Amraei, R. \& Rahimi, N. COVID-19, Renin-Angiotensin System and Endothelial Dysfunction. Cells 9, https://doi.org/10.3390/ cells9071652 (2020).

31. Mone, P. et al. miR-24 Targets the Transmembrane Glycoprotein Neuropilin-1 in Human Brain Microvascular Endothelial Cells. Noncoding RNA 7, https://doi.org/10.3390/ncrna7010009 (2021).

32. Nagele, M. P., Haubner, B., Tanner, F. C., Ruschitzka, F. \& Flammer, A. J. Endothelial dysfunction in COVID-19: Current findings and therapeutic implications. Atherosclerosis 314, 58-62. https://doi.org/10.1016/j.atherosclerosis.2020.10.014 (2020).

\section{Authors Contribution}

L.S., L.Q., L.W., and H.H. contributed to the conception and design of the study, the data analysis, the data interpretation, the manuscript drafting, and the critical revision of the manuscript; L.D., Y.X., Y.L., J.J., X.L. contributed to the data analysis, the data interpretation, the manuscript drafting, and the critical revision of the manuscript; D.W.W., B.H., N.Z. contributed to the data interpretation, the manuscript drafting, and the critical revision of the manuscript.

\section{Funding}

This study was funded by the National Natural Science Foundation of China to L. Shen (81900308), N. Zhou (82070316), and the Informatization Development Project of Shanghai Municipal Commission of Economy and Informatization to L. Shen (202002009).

\section{Competing interests}

The authors declare no competing interests.

\section{Additional information}

Correspondence and requests for materials should be addressed to B.H. or N.Z.

Reprints and permissions information is available at www.nature.com/reprints.

Publisher's note Springer Nature remains neutral with regard to jurisdictional claims in published maps and institutional affiliations. 
(c) (i) Open Access This article is licensed under a Creative Commons Attribution 4.0 International cc) License, which permits use, sharing, adaptation, distribution and reproduction in any medium or format, as long as you give appropriate credit to the original author(s) and the source, provide a link to the Creative Commons licence, and indicate if changes were made. The images or other third party material in this article are included in the article's Creative Commons licence, unless indicated otherwise in a credit line to the material. If material is not included in the article's Creative Commons licence and your intended use is not permitted by statutory regulation or exceeds the permitted use, you will need to obtain permission directly from the copyright holder. To view a copy of this licence, visit http://creativecommons.org/licenses/by/4.0/.

(C) The Author(s) 2021 